\title{
A case of IgA nephropathy with deep venous thrombosis in the mesentery and lower extremities
}

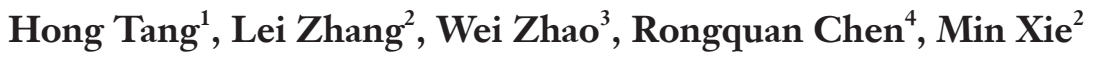 \\ ${ }^{1}$ Department of Pathology, ${ }^{2}$ Department of Imaging, ${ }^{3}$ Department of Vascular Surgery, ${ }^{4}$ Department of Nephrology, The Second People's Hospital \\ of Wuxi, Wuxi 214002, China
}

Correspondence to: Min Xie. Department of Radiology, the Second People's Hospital of Wuxi, 68 Zhongshan Road, Wuxi 214002, China. Email: xiesheng68@aliyun.com.

Submitted Aug 09, 2018. Accepted for publication Nov 07, 2018.

doi: 10.21037/qims.2018.11.05

View this article at: http://dx.doi.org/10.21037/qims.2018.11.05

\section{Introduction}

IgA nephropathy (Berger disease) is a disorder in which there is prominent IgA deposition in the glomerular mesangial area, and is the most common type of primary glomerulonephritis (1), receiving more and more attention (2-8). With diverse clinical manifestations, it has its own characteristic type of glomerular mesangial lesions (9). IgA nephropathy accounts for $45.2-58.2 \%$ of primary glomerulonephritis in China and $1 / 3$ of cases in other countries in Asia, as well as 20\% in Europe and 10\% in the United States. This significant difference in prevalence may be caused by differences in the indications for a renal biopsy between Asia and the United States. IgA nephropathy is associated with an increased risk of deep venous thrombosis (DVT) (10), mostly involving the renal veins and the lower extremities, and sometimes the portal vein and vena cava. Superior mesenteric vein thrombosis is rare. We present a case of IgA nephropathy with deep venous thrombosis of the superior mesenteric vein and both lower extremities. The patient gave informed consent according to the World Medical Association Declaration of Helsinki.

\section{Case presentation}

The patient, a 52-year-old male, was diagnosed as having DVT of the lower extremities in another hospital because of a 10-day history of swelling, pain, and restricted range of motion of the lower extremities. He was transferred to the Department of Vascular Surgery in our hospital for further evaluation. The physical examination showed non- pitting edema and pain involving both lower extremities slightly relieved with elevation, and obvious pain while walking. On the second hospital day, the patient had gradually increasing abdominal pain and occasional diarrhea, accompanied by fever up to $39^{\circ} \mathrm{C}$. There was tenderness and mild guarding in the umbilical area, with no rebound tenderness, and no hyperactive bowel sounds. The laboratory and imaging results: blood: WBC $17.6 \times 10^{9} / \mathrm{L}, 80 \%$ neutrophils; blood coagulation: fibrin (origin) degradation product: $7.90 \mu \mathrm{g} / \mathrm{mL}$; D-dimer: $1.20 \mu \mathrm{g} / \mathrm{mL}$, fibrinogen concentration: $14.02 \mu \mathrm{mol} / \mathrm{L}$ (normal range, 5.8-11.8 $\mu \mathrm{mol} / \mathrm{L}$ ), prothrombin time: $15.2 \mathrm{~s}$ (normal range, 11-13 s), PT normalized ratio: 1.3; globulin ratio: 0.96 , albumin: $27.4 \mathrm{~g} / \mathrm{L}$, alanine aminotransferase: $52.4 \mathrm{U} / \mathrm{L}$; review of blood routine examination: WBC: $26.5 \times 10^{9} / \mathrm{L}, 96$; urinalysis: occult blood: $10 / \mu \mathrm{L} 1+$, leukocytes: $28.8 / \mu \mathrm{L}$, crystallization examination: $0.1 / \mu \mathrm{L}$; stool routine examination: color: yellow, occult blood: positive, qualitative state: semi-liquid. An ultrasound (US) of the lower extremities (Figure 1) was positive for DVT in the muscular calf veins; a contrast-enhanced CT of the abdomen (Figure 2) suggested superior mesenteric vein thrombosis and intestinal necrosis. In the evening of the same day, he was transferred to a higher-level hospital. After treatment with anticoagulation, antibiotics for intestinal necrosis detumescence and detumescence and rehydration for $>20$ days, his condition was improved, with no abdominal pain, and improvement of the swelling and pain in both lower extremities. However, when he returned to the Department of Vascular Surgery of our hospital 


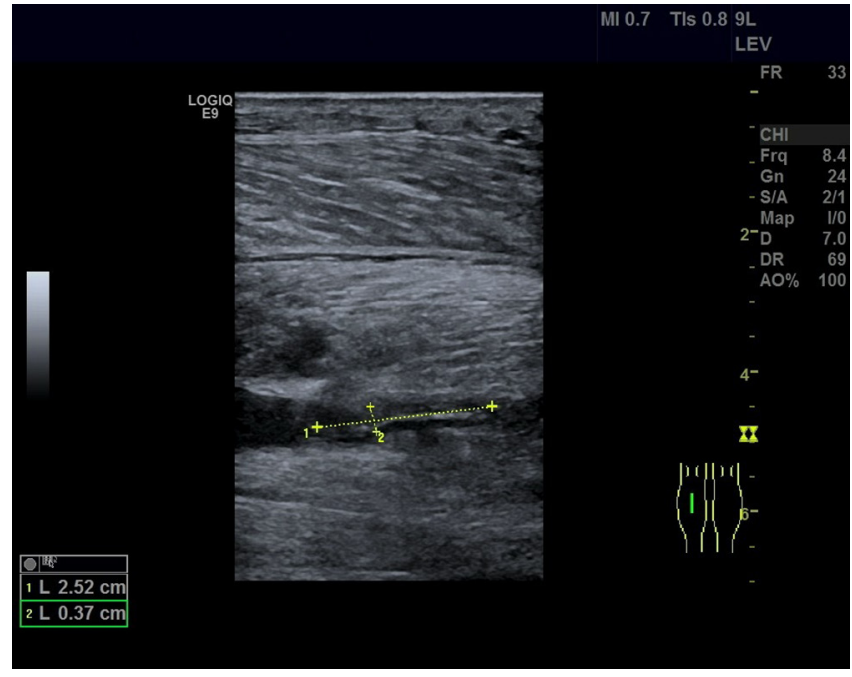

Figure 1 Ultrasound shows leg intermuscular vein ectasia in lower extremities with scattered thrombus.

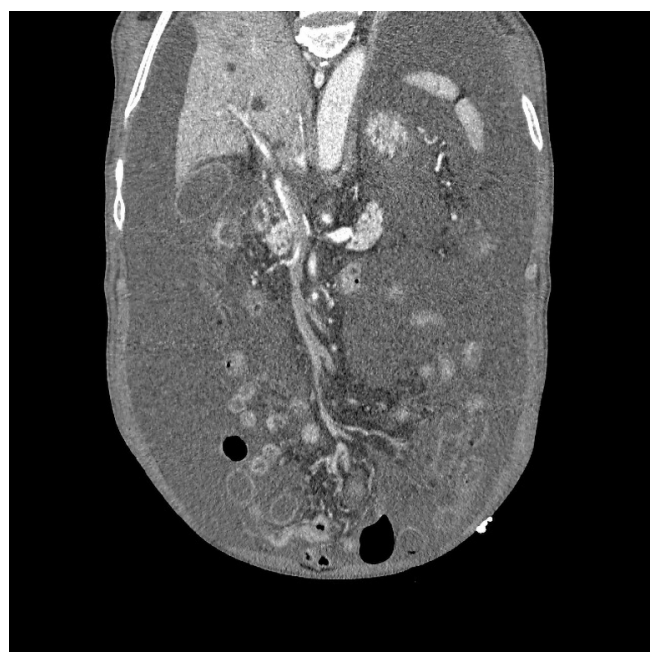

Figure 2 Contrast-enhanced CT with coronal reconstruction shows superior mesenteric vein thrombosis.

to continue the treatment, abdominal pain, diarrhea, and swelling in both lower extremities reappeared. Membranous nephropathy was suspected. He was transferred to the Department of Nephrology. His GFR: left kidney $22.02 \mathrm{~mL} / \mathrm{min}$; right kidney: $24.23 \mathrm{~mL} / \mathrm{min}$ (normal value is $40-60 \mathrm{~mL} / \mathrm{min}$ ). A renal biopsy was performed, with three core samples acquired. Global sclerosis appeared in one glomerulus, spherical cellular crescents were found in three glomeruli, and segmental cellular crescents were found in eight glomeruli. The glomerular capillary loop was

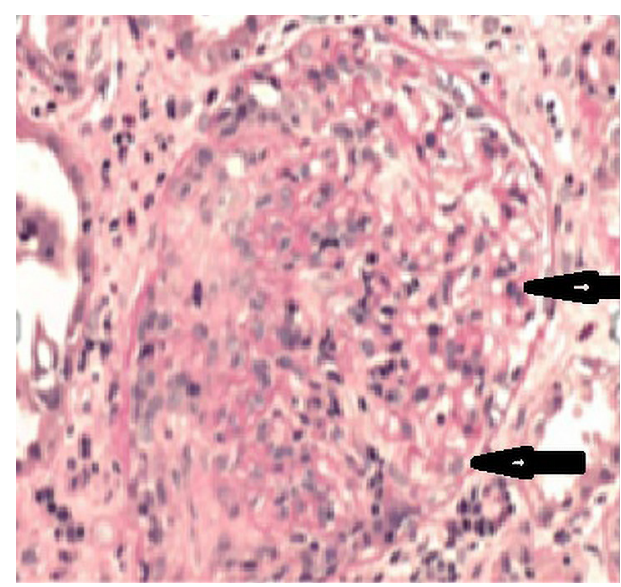

Figure 3 PAS staining $(\times 400)$ shows negative PLA2R. The arrows display mild glomerular mesangial matrix hyperplasia.

obstructed under pressure and was necrotic. The remaining glomeruli were normal in size, with about $80-100$ cells/ glomerulus, 2-3 mesangial cells/mesangial area (slight elevation), mild mesangial matrix hyperplasia, opening of capillary loops, normal basement membranes, and renal capsular adhesions involving some glomeruli. Masson staining was negative. Severe tubulointerstitial lesions were noted, with unclear tubular structures, diffuse tubular epithelial cell edema and degeneration, and necrosis and detachment in certain cells. Protein casts were seen, and there was diffuse infiltration of the interstitium by neutrophils, lymphocytes, and eosinophils. The arteriolar walls were thickened slightly, with intimal hyperplasia. No hyaline degeneration was seen. No thrombosis was seen in the vessel lumen, and no obvious inflammatory cell infiltration was seen in the vessel wall. Immune-combined Periodic Acid-Schiff stain (PAS) staining (Figure 3) and Periodic Acid-Silver Methenamine stain (PASM) staining (Figure 4) suggested that PLA2R was negative and Ig isoforms were negative. Under electron microscopy, there was glomerular capillary loop mesangial matrix hyperplasia, electrondense deposits in the mesangial area (arrow, Figure 5), an even basement membrane, no obvious electron-dense deposits on the epithelial medial or endothelial sides of the basement membrane, and foot process fusion of epithelial cells and cytoplasmic shedding. Necrosis and exfoliation were seen in certain renal tubular epithelial cells, but no electron-dense deposits were found around the tubular basement membrane. Combined with immunofluorescence findings (Figure 6), we diagnosed IgA nephropathy (mesangial proliferative glomerulonephritis with crescent 


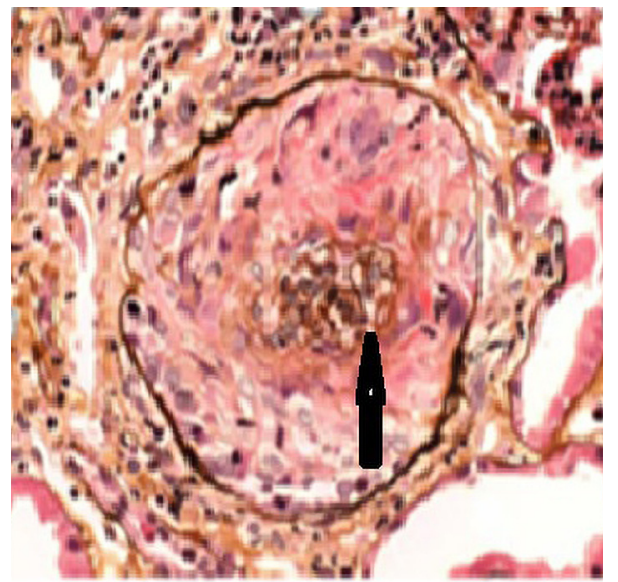

Figure 4 PASM staining $(\times 400)$ shows negative Ig subtype. The arrow displays glomerular sclerosis.

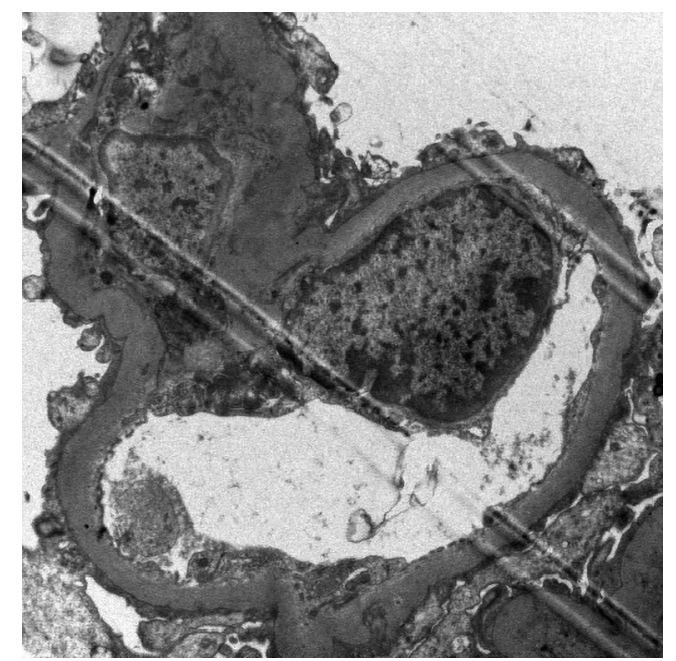

Figure 5 Electron microscopy shows there was glomerular capillary loop mesangial matrix hyperplasia, electron-dense deposits in the mesangial area, but no obvious electron-dense deposits on the epithelial medial and endothelial sides of the basement membrane, and foot process fusion of epithelial cells and cytoplasmic shedding. Necrosis and exfoliation were seen in certain renal tubular epithelial cells.

formation), Hass IV type, M0E0S0T2 (Oxford, 2009) (total number of glomeruli: 15, endothelial cell proliferation: 0 , crescent: 11, global sclerosis: 1, and segmental sclerosis: 0 ).

The patient was administered a single pulse of $500 \mathrm{mg}$ methylprednisolone, and calcium supplements and gastrointestinal protection were given for symptomatic

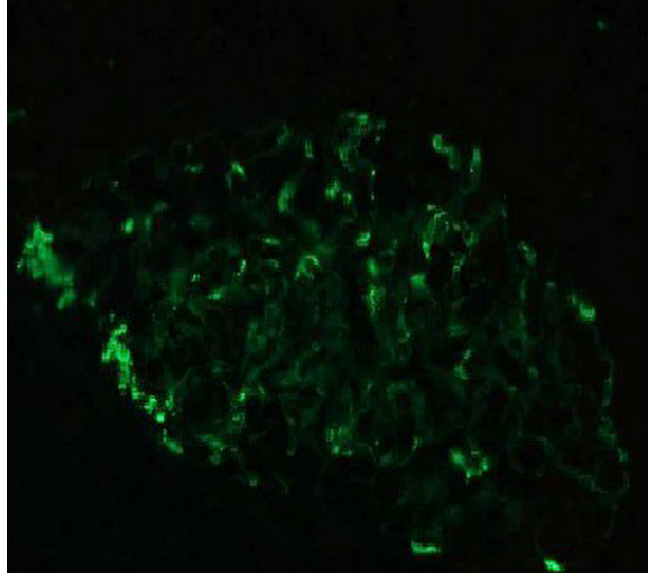

Figure 6 Immunofluorescence $(\times 400)$ shows IgA nephropathy. IgA and $\mathrm{C} 3$ are present in the mesangial area as massive diffuse fluorescent deposits.

supportive treatment. After 3 days, he was significantly improved. He was switched to methylprednisolone tablets $52 \mathrm{mg} /$ day. The patient was discharged after 2 days and was followed as an outpatient, with regular adjustments of the methylprednisolone dose.

\section{Discussion}

\section{Clinical diagnostic signs of IgA nephropathy}

The clinical manifestations of IgA nephropathy mainly include mild painless hematuria and/or proteinuria, or signs of more serious renal damage, such as acute and chronic renal failure, in which the clinically most common feature is microscopic hematuria with or without proteinuria (11-14). Approximately $40-50 \%$ of patients show paroxysmal hematuria; most patients have intermittent or persistent mild to moderate proteinuria, and only about $5 \%$ of patients have proteinuria at nephrotic syndrome levels (15). The incidence of nephrotic syndrome in children and adolescents is significantly higher. The Clinical Guidelines of the Chinese Medical Association for Kidney Diseases (16) point out that although there is no characteristic pattern in the clinical manifestations and laboratory examinations of IgA nephropathy, IgA nephropathy should be suspected if the following symptoms occur: (I) upper respiratory tract infection or tonsillitis attacks with gross hematuria at the same time for at least 1 week, disappearing or decreasing after infection control; (II) hematuria with or without 
proteinuria; (III) high serum IgA levels.

\section{Key points for pathological diagnosis of IgA nephropathy}

IgA nephropathy is a pathologic diagnosis requiring a renal biopsy. Under light microscopy, the most prominent feature of IgA nephropathy is the widening of the mesangial area that results from the proliferation of mesangial cells and mesangial matrix. Immunofluorescence examination shows that a large amount of IgA is present in the mesangial area in the form of massive or granular diffuse deposits, which may be accompanied by the deposition of IgG and IgM. Sometimes, the deposits may extend to the capillary wall. The vast majority of cases are associated with $\mathrm{C} 3$ deposition and IgG and IgM deposition of the same magnitude as the IgA distribution, but the $\mathrm{C} 1 \mathrm{q}$ and $\mathrm{C} 4$ are negative. Masson staining shows that the red IgA, IgG, and IgM deposits are mainly distributed in the mesangial area, mostly in spot or plaque shape, occasionally in short linear collections, and are accompanied by enlargement of the blue mesangial area, namely, increased matrix. Electron microscopy electron-dense deposits in all mesangial areas, indicating that the lesions are diffuse, and a small number of electrondense material deposits can be seen occasionally under the endothelium or epithelium, especially in critically ill patients. The Kidney Disease: Improving Global Outcomes (KDIGO) guidelines point out that all patients with renal biopsy-proven IgA nephropathy should undergo secondary pathogenesis identification (17-21). Common secondary glomerular diseases including lupus nephritis, HenochSchönleinpurpura nephritis, hepatitis B nephritis, as well as liver cirrhosis, inflammatory bowel disease, and human immunodeficiency virus (HIV) nephropathy should be identifiable by the glomerular IgA deposits.

The study of pathological grading for IgA nephropathy has developed from more emphasis on quantitative classification, usually including Lee's grading and Haas's grading, which divide it into grades I, II, III, IV and V from light to severe based on the severities of glomerular and tubulointerstitial lesions. In 2009, the International IgA Nephropathy Network and the Renal Pathology Society jointly published the Oxford Classification of IgA Nephropathy, focusing on levels of mesangial cell proliferation $(\mathrm{M})$, segmental glomerulosclerosis (S), intra capillary cell hyperplasia (E) and tubular atrophy/interstitial fibrosis (T). Through a series of evidence-based studies, the Oxford Classification Group suggested that renal M, S, E and $\mathrm{T}$ lesions detected on renal biopsy are closely related to indicators such as 24-hour urinary protein quantification, blood pressure levels, and glomerular filtration rate (GFR), and can be used to evaluate the prognosis of patients with IgA nephropathy (22). Strictly formulated with full consideration for repeatability, Oxford's pathological classification system is currently the most rigorous and scientific classification method and is considered to have good clinical utility.

\section{Mechanism of IgA nephropathy with thrombosis}

Venous thrombosis is mainly caused by aggregation of fibrin and red blood cells, and the main components of arterial thrombosis are platelets and a small amount of fibrin. Venous thrombosis is more clinically common. Factors that are currently recognized to promote thrombosis include vascular endothelial cell damage, hypercoagulable state, and hemodynamic changes $(23,24)$. Immune complexes and autoantibodies in patients with anti-IgA nephropathy can cause vascular endothelial damage with subsequent collagen exposure and that can initiate endogenous and exogenous coagulation pathways. Exogenous tissue factor plays an important role in the formation of a hypercoagulable state that is the result of multiple pathophysiological changes (25). IgA nephropathy complicated by DVT in both lower extremities is rare, and simultaneous mesenteric venous thrombosis is rarer (26). Studies found that IgA nephropathy causes significant microvascular damage (27-30), and IgA patients are more prone to arteriosclerosis than healthy people of the same age group.

\section{Treatment progress in IgA nephropathy}

An effective individualized treatment plan is developed according to the histopathological type and grade found in the renal biopsy. Obvious inflammatory cell infiltration, proliferation of mesangial cells, and formation of cellular crescents are indications for using immunosuppressive agents. For patients with simple hematuria IgA nephropathy, the KDIGO guidelines do not recommend tonsillectomy or antiplatelet drug treatment due to insufficient evidence. Whether to adopt more aggressive initial immunosuppressive treatment regimens for patients with IgA nephropathy and the setting of treatment timing and program for severe IgA nephropathy patients with initial poor renal function is subject to the development of large-scale randomized controlled trials and large data 
analyses.

In summary, the clinical manifestations of IgA nephropathy are diverse, such as extensive DVT, abdominal pain, anemia, and proteinuria in this patient. The conditions were treatment-resistant. After a multi-disciplinary consultation, the renal physician suggested the possibility of membranous nephropathy, so a renal biopsy was performed (31), and accurate pathological grading, definitive diagnosis of IgA nephropathy, and effective treatment followed (32-34). It is difficult to diagnose or clarify IgA nephropathy, so multi-disciplinary consultations should be organized. Renal biopsy is necessary for the pathological diagnosis, and CT angiography and MR angiography are the gold standards for the diagnosis of thromboembolism in CT and MRI examinations.

\section{Acknowledgements}

We thank Libby Cone, MD, MA, from Liwen Bianji, Edanz Group China (www.liwenbianji.cn/ac) for editing a draft of this manuscript.

\section{Footnote}

Conflicts of Interest: The authors have no conflicts of interest to declare.

Informed Consent: The patient gave informed consent according to the World Medical Association Declaration of Helsinki.

\section{References}

1. Espinosa M, Ortega R, Gomez-Carrasco JM, LopezRubio F, Lopez-Andreu M. Mesangial C4d deposition: a new prognostic factor in IgA nephropathy. Nephrol Dial Transplant 2009;24:886-91.

2. Cheng IK, Chan KW, Chan MK. Mesangial IgA nephropathy with steroid-responsive nephrotic syndrome: disappearance of mesangial IgA deposits following steroidinduced remission. Am J Kidney Dis 1989;14:361-64.

3. Gallego N, Gonzalo A, Mampaso F, Ortuno J. Steroiddependent nephrotic syndrome with minimal glomerular changes and mesangial IgA deposits. Child Nephrol Urol 1988-1989;9:98-100.

4. Clive DM, Galvanek EG, Silva FG. Mesangial immunoglobulin A deposits in minimal change nephrotic syndrome: a report of an older patient and review of the literature. Am J Nephrol 1990;10:31-6.

5. Westhoff TH, Waldherr R, Loddenkemper C, Ries W, Zidek W, van der Giet M. Mesangial IgA deposition in minimal change nephrotic syndrome: coincidence of different entities or variant of minimal change disease? Clin Nephrol 2006;65:203-7.

6. Wang J, Juan J, Huang Q, Zeng C, Liu Z. Corticosteroid therapy in IgA nephropathy with minimal changelike lesions: a single-centre cohort study. Nephrol Dial Transplant 2013;28:2339-45.

7. Herlitz LC, Bomback AS, Stokes MB, Radhakrishnan J, Daqati VD, Markowitz GS. IgA nephropathy with minimal change disease. Clin J Am Soc Nephrol 2014;9:1033-9.

8. Lai KN, Lai FM, Ho CP, Chan KW. Corticosteroid therapy in IgA nephropathy with nephrotic syndrome: a long-term controlled trial. Clin Nephrol 1986;26:174-80.

9. Donadio JV Jr, Grande JP. Immunoglobulin A nephropathy: a clinical perspective. J Am Soc Nephrol 1997;8:1324-32.

10. Kim SM, Moon KC, Oh KH, Joo KW, Kim YS, Ahn C, Han JS, Kim S. Clinicopathologic characteristics of IgA nephropathy with steroid-responsive nephrotic syndrome. J Korean Med Sci 2009;24:S44-9.

11. Wyatt RJ, Julian BA. IgA nephropathy. N Engl J Med 2013;368:2402-14.

12. Gutiérrez E, Zamora I, Ballarin JA, Arce Y, Jimenez S, Quereda C, Olea T, Martinez-Ara J, Seqarra A, Bernis C, Garcia A, Goicoechea M, Garcia de Vinuesa S, RojasRivera J, Praqa M; Grupo de Estudio de Enfermedades Glomerulares de la Sociedad Espanola de Nefroloqia (GLOSEN). Long-term outcomes of IgA nephropathy presenting with minimal or no proteinuria. J Am Soc Nephrol 2012;23:1753-60.

13. Barbour SJ, Cattran DC, Kim SJ, Levin A, Wald R, Hladunewich MA, Reich HN. Individuals of Pacific Asian origin with IgA nephropathy have an increased risk of progression to end-stage renal disease. Kidney Int 2013;84:1017-24.

14. Suzuki H, Kiryluk K, Novak J, Moldoveanu Z, Herr AB, Renfrow MB, Wyatt RJ, Scolari F, Mestecky J, Gharavi AG, Julian BA. The pathophysiology of IgA nephropathy. J Am Soc Nephrol 2011;22:1795-803.

15. Haas M. IgA nephropathy histologically resembling focalsegmental glomerulosclerosis: a clinicopathologic study of 18 cases. Am J Kidney Dis 1996;28:365-71.

16. Barratt J, Feehally J. IgA nephropathy. J Am Soc Nephrol 2005;16:2088-97.

17. KDIGOBoardMembers. KDIGO clinical practice 
guideline for glomerulonephritis. Kidney Int Suppl 2012;2:139-274.

18. Hashimoto T, Toya Y, Kihara M, Yabana M, Inayama Y, Tanaka K, Iwatsubo K, Yanaqi M, Oshikawa J, Kokuho T, Kuji T, Yoshida S, Tamura K, Umemura S. Behect's disease complicated by IgA nephropathy with nephrotic syndrome. Clin Exp Nephrol 2008;12:224-7.

19. Cho SB, Kim J, Kang SW, Yoo TH, Zheng Z, Cho S, Lee HS, Banq D. Renal manifestations in 2007 Korean patients with Behect's disease. Yonsei Med J 2013;54:189-96.

20. Akutsu Y, Itami N, Tanaka M, Kusunoki Y, Tochimaru H, Takekoshi Y. IgA nephritis in Behect's disease: case report and review of the literature. Clin Nephrol 1990;34:52-5.

21. Ardalan MR, Sadreddini S, Noshad H, Ebrahimi A, Molaeefard M, Somi MH, Shoja MM. Renal involvement in Behect' disease. Saudi J Kidney Dis Transpl 2009;20:618-22.

22. Reich HN, Troyanov S, Scholey JW, Cattran DC. Remission of proteinuria improves prognosis in IgAN. J Am Soc Nephrol 2007;18:3177-83.

23. BarbanoB, Gigante A, Amoroso A, Cianci R. Thrombosis in nephrotic syndrome. Semin Thromb Hemost 2013;39:469-76.

24. Jain P, Sandeep N. Portal vein thrombosis: etiology and clinical outcome of cirrhosis and malignancy-related non-cirrhotic, non-tumoral extrahepatic portal venous obstruction. World J Gastroenterol 2007;13:5288-9.

25. Mirrakhimov AE, Ali AM, Barbaryan A, Prueksaritanond S, Hussain N. Primary nephrotic syndrome in adults as a risk factor for pulmonary embolism: an up-to-date review of

Cite this article as: Tang H, Zhang L, Zhao W, Chen R, Xie M. A case of IgA nephropathy with deep venous thrombosis in the mesentery and lower extremities. Quant Imaging Med Surg 2018;8(11):1123-1128. doi: 10.21037/qims.2018.11.05 the literature. Int J Nephrol 2014;2014:916760.

26. Kumar S, Sarr MG, Kamath PS. Mesenteric venous thrombosis. N Engl J Med 2001;345:1683-88.

27. Barbour T, Johnson S, Cohney S, Hughes P. Thrombotic microangiopathy and associated renal disorders. Nephrol Dial Transplant 2012;27:2673-85.

28. Sagripanti A, Barsotti G. Hypercoagulability, intraglomerular coagulation, and thromboembolism in nephrotic syndrome. Nephron 1995;70:271.

29. Haas M. Histologic subclassification of IgA nephropathy: a clinicopathologic study of 244 cases. Am J Kidney Dis 1997;29:829-42.

30. Shigematsu H. Histologic grading and staging of IgA nephropathy. Pathol Int 1997;47:194-202.

31. Akpolat T, Dilek M, Aksu K, Keser G, Toprak O, Cirit M, Oquz Y. Taskapan H, Adibelli Z, Akar H, Tokqoz B, Arici M, Celiker H, Diri B, Akpolat I. Renal Behect' disease: an update. Semin Arthritis Rheum 2008;38:241-8.

32. Sogaard KK, Astrup LB, Vilstrup H, Gronbaek H. Portal vein thrombosis; risk factors, clinical presentation and treatment. BMC Gastroenterol 2007;7:34.

33. Malkowski P, Pawlak J, Michalowicz B, Sczzerban J, Wroblewski T, Leowska E, Krawczyk M. Thrombolytic treatment of portal thrombosis. Hepatogastroenterology 2003,50:2098-100.

34. Uflacker R. Applications of percutaneous mechanical thrombectomy in transjugular intrahepatic portosystemic shunt and portal vein thrombosis. Tech Vasc Interv Radiol 2003;6:59-69. 